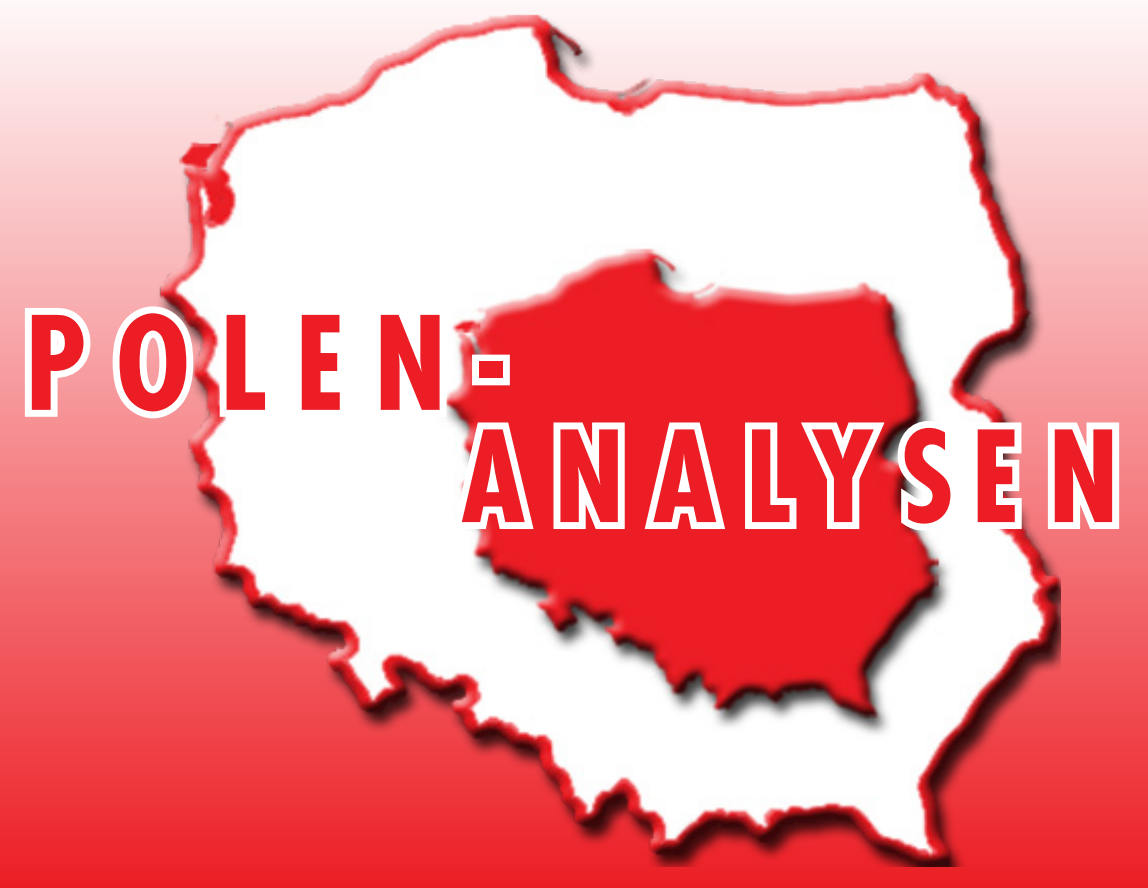

www.laender-analysen.de/polen

\title{
DIE POLEN UND DIE ENERGIEWENDE
}

ANALYSE

Die Polen und die Energiewende

Michał Olszewski, Krakau

- TABELLEN UND GRAFIKEN ZUM TEXT

Der Energiemix im Vergleich und Erdöl/Erdgas nach Herkunftsländern

\section{CHRONIK}

Vom 19. März bis zum 15. April 2013

\section{VERANSTALTUNGSANKÜNDIGUNG}

Dritte Tagung Deutsche Polenforschung

Gießen, 20.-22. März 2014

Wissen, Verstehen, Übersetzen: Nachbarn im Dialog 


\title{
Die Polen und die Energiewende
}

\author{
Michał Olszewski, Krakau
}

\section{Zusammenfassung}

Der Autor vergleicht die Diskussion und die konkreten Schritte zu der von der Politik postulierten Energiewende in Deutschland mit der Situation in Polen. Während er die Umsetzung in Deutschland von einer breiten Mehrheit getragen sieht, stellt er für Polen große Vorbehalte gegenüber erneuerbaren Energien und einer grundlegenden Umstrukturierung des Energiesektors fest. Zurückzuführen sei die Zurückhaltung weniger auf finanzielle Gründe als auf das mangelnde Verständnis von der umweltpolitischen Notwendigkeit und auf den Einfluss von traditionellen energiepolitischen Lobbygruppen. Der Autor behauptet einen Mangel an Einsicht, dass es keine Alternative zu dem von der EU befürworteten Energiekurs gibt, und an Mut, sich in Polen den mit einer energiepolitischen Wende einhergehenden Reformen zu stellen.

$\mathrm{D}$ er im Süden Berlins in der Nähe des Flughafens Tempelhof gelegene EUREF-Campus sieht aus polnischer Perspektive - leider - wie ein fernliegendes futuristisches Projekt aus. Auf einem Gelände rund um den Berlin Gasometer, eingebettet zwischen Bahnlinie und Wohnsiedlung, entsteht im Stadtteil Schöneberg ein Quartier, das ein Beispiel für die neue Herangehensweise der Deutschen an die Energiegewinnung sein soll. Langfristig sollen hier 25 Gebäude entstehen, in die Forschungsinstitute, Behörden für Energiepolitik und Büros großer Firmen einziehen sollen. Vorgesehen ist, dass dieses Terrain gleichzeitig sowohl Arbeitsplatz für Bürotätigkeiten als auch Ort der Energieproduktion sein soll. Die Arbeitnehmer von InnoZ (einer Einrichtung, die u. a. von der Deutschen Bahn finanziert wird und sich mit der Produktion von Energie und Gas sowie der Suche nach neuen Lösungen im Bereich des Stadtverkehrs befasst), die durch die Anlage führen, berichten, dass der Campus im Sommer 2012 zum ersten Mal energetische Autarkie erreicht hatte.

Auf den Dächern befinden sich Photovoltaikanlagen und lautlos arbeitende Windturbinen der neuesten Generation, in einem der Gebäude wandelt ein hocheffektiver Stirlingmotor Gas in Strom und Wärme um. Das Gas stammt aus Zisternen der Biogasanlage. Viel Grün, Ladestationen für Elektroautos. Damit gibt es natürlich ein Problem: Man lädt fünf Stunden, um knapp mehr als 100 Kilometer zu fahren, und die Batterie ist so groß, dass ein Mini Morris zum Auto für zwei Personen wird. Oder anders gefragt: Wie »betankt« man einen Bus mit Energie?

Solche Schwierigkeiten scheinen hier niemanden zu erschrecken. Die Batterien kann man ja in speziellen Garagen wechseln. Busse? Die Arbeiten zur induktiven Energieversorgung, dank deren Kabel überflüssig werden, dauern an. Die Firmen, die bereits auf dem Campus ansässig sind, haben das Projekt "BeMobility - Berlin elektroMobil« ins Leben gerufen. Kauft der Kunde eine Monatskarte für 78 Euro, ist er berechtigt, den öffentlichen Verkehr kostenlos zu nutzen; nimmt er ein städtisches Leihfahrrad in Anspruch, hat er jedes Mal 30 Freiminuten, und er hat die Möglichkeit, Elektroautos im Carsharing zu nutzen. Es entstehen Telefonapps, die bei Bedarf Autos in der Nähe ausfindig machen, die geliehen werden können.

Der Gast aus Polen könnte den Vorwurf formulieren, dass der EUREF-Campus eine Laune sei, die sich die reichen Deutschen leisten können, aber nicht die armen Polen. Die Höhe der Ausgaben ist tatsächlich unglaublich: Die Gesamtkosten für die Investition belaufen sich auf zirka 600 Millionen Euro.

\section{Der polnische Energiemix: vor allem Kohle}

Ein Problem besteht ebenfalls - oder vielleicht vor allem in der Mentalität. Geld ist eine wesentliche Barriere, aber das ist es nicht, was EUREF aus polnischer Perspektive nicht nachvollziehbar oder sogar verdächtig macht, so wie übrigens die gesamte Energiewende. Dieses umfangreiche gesellschaftlich-technologische Projekt, das das verbinden soll, was dem Anschein nach unmöglich ist, nämlich sowohl die Sicherung der Position Deutschlands als weltweit führend in Sachen Technologieentwicklung als auch die Bewahrung Deutschlands vor der Abwanderung der Schwerindustrie in andere Länder, wird in Polen mit tiefem Misstrauen wahrgenommen. Mehr noch: Je mehr Zeit seit der Unterzeichnung des Klimapakets durch Polen im Jahr 2008 vergeht, desto größer wird das Misstrauen. Gewerkschaften, Bergarbeiter, Politiker des linken und des rechten Spektrums, der Ministerpräsident, der Umweltminister und viele Bürger - die Mehrheit ist überzeugt, dass die Abkehr der Wirtschaft vom Energieträger Kohle für Polen nur eine Niederlage und die Verstärkung sozialer Probleme bedeuten kann. Daher tauchen immer häufiger Aufrufe auf, die europäischen Klimaverhandlungen zu kippen - gänzlich unverantwortliche Aufrufe, zieht man in Erwägung, dass Umweltschutz und der Kampf mit der globalen Erwärmung Fundamente der europäischen Politik sind. 
Die Energiewende in trockenen Zahlen bedeutet: Bis zum Jahr 2050 will Deutschland den Ausstoß von Treibhausgasen um 80 Prozent im Vergleich zu 1990 verringern sowie den Energiebedarf um 20 Prozent im Vergleich zu 1990, wobei die Industrieproduktion nicht reduziert werden soll. Dieses Projekt ist außergewöhnlich kostenintensiv und kompliziert, es verbindet sich mit unvermeidlichen Kostenerhöhungen für Energie, aber grundsätzlich wird es von allen großen Akteuren auf der politischen Bühne befürwortet. Dies ändern nicht einmal Gerüchte über die Größe der notwendigen Investitionen - in den kommenden acht Jahren könnte Deutschland über 150 Milliarden Euro für die Modernisierung des Energienetzes und verschiedene Formen der Unterstützung für erneuerbare Energiequellen ausgeben. Das Fundament der polnischen Wirtschaftspolitik ist dagegen ein vollkommen anderes. Es ist unantastbar und nichts weist darauf hin, dass es sich in den nächsten Jahren verändern wird. Sein Name ist Kohle. Obwohl die Kohlevorkommen in Polen bis zum Jahr 2035 erschöpft sein werden, wovor ein offizieller Bericht der Obersten Kontrollkammer (Najwyższa Izba Kontroli) warnt, obwohl der Kohleimport wächst, soll Kohle im polnischen Energiemix in den nächsten Jahrzehnten die entscheidende Rolle spielen. Der gegenwärtige Anteil von zirka 90 Prozent wird möglicherweise bis 2030 auf 56 Prozent fallen.

\section{Wo bleibt die Vision?}

Dabei soll hier deutlich gesagt werden, dass Polen die letzten 20 Jahre im Bereich des breit verstandenen Umweltschutzes nicht verschlafen hat. Die Verpflichtungen des Kyoto-Protokolls, das eine Reduzierung der Treibhausgase um sechs Prozent zwischen 1988 und 2012 festlegte, haben wir mit großem Überschuss übererfüllt - um 28 Prozent, sowohl dank der Umstrukturierungen in der Industrie als auch der Rezession Anfang der 1990er Jahre, die einen großen Teil der emissionsintensiven Industrie aus dem Markt drängte. Gleichzeitig begingen die sich ablösenden Regierungen aber eine fatale Unterlassungssünde: Es fand sich kein Politiker, der die Last einer wirksamen Reform des Strom- und Bergbausektors auf sich genommen hätte. Diese hätte unvermeidliche und womöglich gewaltsame Proteste der Bergarbeiter zur Folge sowie eine Umverteilung der Zuflüsse zum Staatshaushalt (davon später mehr). In der Folge begann Polen die ambitionierte Klimapolitik der EU mit einer Bürde umzusetzen, deren Gewicht umso spürbarer wird, je mehr wir uns dessen bewusst werden, dass der Vertrag von Dezember 2008 das Schicksal der Gesamtgesellschaft bestimmt und es nicht möglich sein wird, zu entkommen. Deshalb sollten Orte wie der EUREF-Campus oder das neue und zugleich schöne und unglaublich sparsame Gebäude der lokalen Behörden in Eberswalde bei Berlin in Polen besser nicht laut genannt werden. Deutschland hat strenge Energiesparnormen, Unternehmen, die sich mit Photovoltaik und Sonnenenergie befassen, grüne Dächer und erstaunliche Einfälle, wie man noch sparen könnte (beispielsweise indem die Wärme des Serverraums genutzt wird, um das Gebäude zu heizen wie im Sitz der Heinrich Böll Stiftung). Polen hat vor allem Bergarbeiter, die viel mehr als der Landesdurchschnitt verdienen und schnell eine hohe Rente bekommen - mit zirka 6 Milliarden Zloty jährlich großzügig von der Sozialversicherungsanstalt (Zakład Ubezpieczeń Społecznych - ZUS) unterstützt. Es hat eine der energieintensivsten Wirtschaften in der EU und ein Durchleitungsnetz in einem fatalen Zustand. 40 Prozent der Energiekraftwerke arbeiten schon länger als 40 Jahre, was nicht nur ein hohes Unfallrisiko bedeutet, sondern auch eine geringe Effektivität bei der Energieerzeugung.

Noch etwas unterscheidet uns auf fundamentale Weise. Es wird mir deutlich, wenn ich durch Deutschland fahre, Biogasanlagen und neue Gebäude besichtige und mit Experten spreche, sowohl mit denen, die die Energiewende enthusiastisch begrüßen, als auch mit denen, die sie mit größerer Distanz betrachten. Es scheint, dass es den Deutschen gefällt, Visionen zu haben, es scheint, dass sie nach einer grundsätzlichen Debatte und einer abschließenden Entscheidung das $O b$ zu diskutieren aufgehört haben. Stattdessen denken sie über das Wie nach und machen sich auf den Weg in Richtung des gewählten Ziels. Es ist kein Zufall, dass alle großen Parteien dem generellen Kurs der Wirtschaft in Richtung erneuerbare Energie, Energieeffizienz und einer weiteren Emissionsreduzierung zustimmen. Wenn es um die Umsetzung geht, wird die Diskussion durchaus erbittert geführt, doch habe ich in Deutschland keinen Politiker, Experten oder Investor getroffen, der offen gesagt hätte, dass die EU-Klimapolitik eine Katastrophe sei und man sich schnellstmöglich von ihr verabschieden sollte. Dieser fehlende Protest kann mit dem starken ökologischen Bewusstsein der Deutschen zusammenhängen und ihrem Wohlstand, aber - und hier greife ich wieder die Vision auf - auch mit ihrem Wissen, welche Richtung die EU für die kommenden Jahren eingeschlagen hat, und mit der Fähigkeit, eine langfristige Perspektive zu verfolgen. EUREF ist hier einer der Beweise.

In Polen ist das anders: Hier unterliegt alles einer nicht enden wollenden Verhandlung. Zwar haben wir das Klimapaket unterschrieben, aber man kann es doch kündigen. Zwar gibt es Umweltdirektiven der EU, aber man kann ihre Umsetzung ins polnische Rechtssystem ins Unendliche hinausschieben und darauf zäh- 
len, dass Brüssel es vergisst. Es gibt glaubwürdige Daten zum Einfluss der globalen Erwärmung auf die polnische Wirtschaft, aber nichts hindert den Umwelt- und den Finanzminister daran, offiziell oder inoffiziell diese Glaubwürdigkeit anzuzweifeln. Zwar gibt es ein Ziel, aber ein Ziel kann man ändern.

\section{$\mathrm{Zu}$ innovativ und zu kostspielig, also sinnlos}

Bis zu einem gewissen Grad ist dieses Misstrauen begründet. Seit längerem ist klar, dass das Kyoto-Protokoll nicht umgesetzt wird, und am Horizont ist weit und breit kein Hoffnungsschimmer zu sehen, dass es zu einer neuen Vereinbarung kommen wird. Das Protokoll legt nur für die Industriestaaten verbindliche Reduktionsziele fest (und auch nicht für alle, denn die Vereinigten Staaten haben es nicht ratifiziert). Die großen und dynamischen Wirtschaften von Südkorea, Indien, China oder Brasilien erhöhen ihre Emissionen ohne Einschränkung, der globale Ausstoß von Treibhausgasen steigt und erreicht Höchstwerte in der Geschichte der Messung. Aus dieser Perspektive sehen für Polen, das an die Schwerindustrie gebunden ist, die internen Bemühungen der EU zugunsten einer immer radikaleren Absage an die Kohle so aus, als würde die EU aus der Reihe tanzen. Die Frage, warum nur ein Teil der Welt den Kampf gegen die Klimaveränderung anführen soll, während andere Länder die Anstrengungen zunichtemachen, bleibt bestehen. Die Antwort, dass darüber u. a. historische Entwicklungen entschieden haben, überzeugt hier niemanden.

Ich möchte den Eindruck vermeiden, dass dieser Artikel aus der Perspektive eines kleinen armen Jungen geschrieben wurde, der neidisch auf das Spielzeug seines reichen Cousins schaut. Ich möchte lieber von einer Irritation sprechen. Eine Irritation darüber, dass wir es als Land aus irgendwelchen Gründen nicht einmal versuchen, an die Anführer im technologischen Bereich aufzuschließen, vielmehr entfernt sich dieser immer mehr. Warum sehen deutsche Journalisten, die auf eine Reise durch Polen eingeladen werden, nicht solche Gebäude wie in Eberswalde, warum sehen sie nicht einmal breit angelegte Wärmedämmungsmaßnahmen, die in einem Land mit Gebäuden, die Sieben ähneln, selbstverständlich sein müssten? Sie sehen dies deshalb nicht, weil der Finanzminister den Versuch abbrach, wirksame Vorschriften zur Energieeffizienz einzuführen, da ihn die Perspektive niedriger Einnahmen aus der Akzise für den Verkauf von Energie erschreckt hat. Sprechen wir nicht von Eberswalde - in Polen wurde bisher nicht einmal ein Gesetz zu erneuerbaren Energien verabschiedet, dank dessen der Eigentümer von sogenannten Mikroenergiezellen Energie ins Netz einspeisen dürfte, ohne dass er gleichzeitig verpflichtet wäre, ein wirtschaft- liches Unternehmen zu gründen. Obwohl die Bedingungen für die Entwicklung erneuerbarer Energien in Polen und in Deutschland vergleichbar sind, wird an der Weichsel der Bau von Windkrafträdern als sinnloses Unterfangen beurteilt, das die Landschaft zerstört und sich wirtschaftlich nicht bezahlt macht. Es dominieren die irritierende Strategie der Verzögerung und Verspätungen unausweichlicher Lösungen. Das Schicksal dieses Gesetzes ist in gewissem Sinne symbolisch für die polnischen Überlegungen zu den Berührungspunkten zwischen Umweltschutz und Wirtschaft: Erneuerbare Energiequellen werden von polnischen Politikern und Vertretern der Energiekonzerne als Bedrohung für die Stabilität des Landes, eine Erfindung von Gazprom, einen Anschlag auf die Unabhängigkeit und Traum eines Wahnsinnigen betrachtet.

Vielleicht verbirgt sich hinter dieser Säumigkeit die Überzeugung, dass Polen Schiefergasvorkommen hat, die ein bisschen wie der Hase aus dem Zylinder auf zauberhafte Weise unsere Energieprobleme lösen werden. Solange hier aber noch nichts passiert, bleibt man besser bei der bewährten Kohle, umso mehr, als die öffentliche Meinung nicht allzu wissbegierig ist und Fachleute, die zu überzeugen suchen, dass eine günstigere Energiequelle bisher noch nicht erfunden wurde, von niemandem nach den externen Kosten beispielsweise in Form von Atemwegserkrankungen gefragt werden. Die Verschwörung um die Kohle erinnert mich etwas an eine Geschichte von vor zehn Jahren, als ich in die Stadt Szczucin in der Woiwodschaft Kleinpolen (woj. małopolskie) fuhr. Einige Jahrzehnte lang war dort die landesweit größte Asbestfabrik in Betrieb. In Szczucin starben die Leute massenhaft an Krebs, aber vor dem Tor des abgewickelten Betriebs wurde gefordert, die Produktion des giftigen Stoffes beizubehalten.

Eines der Dogmen, die die polnische Diskussion über die Energiewende und die Klimapolitik im Allgemeinen begleiten, ist ihre vermeintliche Unrentabilität. Die polnische öffentliche Meinung übergeht also fast vollständig den Nutzen, der sich aus diesem umfassenden Infrastrukturprojekt ergibt, und greift dessen schwachen Punkte auf. Für Mariusz Janik von der Tageszeitung "Dziennik Gazety Prawnej« (Nr. 63/2013, „Po rewolucji płoną lasy«/dt.: Nach der Revolution brennen die Wälder) war beispielsweise das Wichtigste, dass die Deutschen anfingen, Holz aus dem Wald zu klauen, weil sie die höheren Heizkostenrechnungen nicht zahlen wollten. "Zwei Jahre nachdem die deutsche Regierung beschlossen hatte, alle Kernkraftwerke vom Netz zu nehmen und auf ökologische Energiequellen umzustellen, versank das Land im Streit. Zwar gibt es Strom im Überfluss, aber es zeigt sich, dass der dramatische Mangel an Leitungsnetzen und die Neuverlegung der- 
selben Proteste wecken, und der Preis für die Lieferung steigt alarmierend. Die grüne Revolution verwandelt sich schrittweise in einen Alptraum«, urteilt der Journalist. Sehr gern werden Signale von steigenden Energiepreisen, geplanten Kohlekraftwerken und, was damit einhergeht, vom Risiko größerer $\mathrm{CO}_{2}$-Emissionen aufgegriffen. Großes Interesse weckt auch die Frage der Abschaltung der Kernkraftwerke - ein Teil der polnischen Kommentatoren ist überzeugt, dass dies hochriskant sei und unvorhersehbare Konsequenzen mit sich bringen werde, was die Klage der drei Energieriesen RWE, E.ON und Vattenfall gegen die deutsche Regierung zu bestätigen scheint, die ihren Gesamtverlust aufgrund eines zu schnellen Rückzugs aus dem Atomprogramm auf 15 Milliarden Euro schätzen. Die meisten Emotionen ruft auf polnischer Seite aber die unkontrollierte Energiezufuhr aus den Windparks in Norddeutschland hervor. Da die deutschen Leitungsnetze nicht mit dem Überschuss zu Spitzenzeiten der Gewinnung zurechtkommen, fließt ein Teil des Windstroms über Polen nach Süddeutschland, was die Möglichkeit blockiert, Strom aus Deutschland nach Polen zu importieren, und außerdem die Kriterien für ein sicheres Funktionieren des Landesweiten Elektroenergetischen Systems (Krajowy System Elektroenergetyczny) überschreitet. Infolgedessen hat der polnische Netzbetreiber Polskie Sieci Energetyczne (PSE S.A.) zusammen mit einem deutschen Netzbetreiber eine Ausschreibung für einen Phasenschiebertransformator getätigt. Unklar ist auch, welchen Einfluss die steigenden Energiepreise auf die Schwerindustrie haben werden. Deutschland will sie bei sich halten, und zwar mit einer Strategie, die der polnischen diametral entgegengesetzt ist - in Polen kann ein energieintensiver Betrieb mit einer Reihe von Erleichterungen und Privilegien rechnen, anders als der Individualkonsument.

\section{Es gibt keine Alternative zu alternativen Energien}

Dies alles entspricht der Wahrheit, allerdings ist dies nicht die ganze Wahrheit. Die Energiewende ist für die deutsche Gesellschaft von enormem Nutzen, aber daran wird in Polen schon weniger gern erinnert. Nach Angaben des Zentrums für Oststudien (Ośrodek Studiów Wschodnich - OSW) in Warschau überstieg die Zahl der Beschäftigten im Sektor erneuerbare Energien in Deutschland im Jahr 2010360.000 Personen, und wenn das Tempo dieser Entwicklung anhält, werden es im Jahr 2020500.000 sein. Zwischen 2000 und 2010 stieg der Export von Photovoltaikkomponenten fast um das Siebenfache und für Windkraftwerke um das Neunfache. Wie sieht Polen vor diesem Hintergrund aus? Aus der Windkraft gewinnen wir bereits 1,5 Mal mehr Energie als Österreich oder Belgien, aber gleichzeitig $18 \mathrm{Mal}$ weniger als Deutschland, wo es doch ähnlich weht wie in Polen. Die Gesamtkapazität der installierten Sonnenkollektoren und Photovoltaikanlagen ist 20 Mal niedriger als bei unserem Nachbarn. Diese Daten gewinnen zusätzlich an Bedeutung, wenn man den tatsächlichen Preisanstieg mit der gleichzeitig steigenden Zustimmung, die Kosten für den Kampf mit der Klimaveränderung zu tragen, kombiniert. Eurostat hat errechnet, dass in den Jahren 2007 bis 2010 der durchschnittliche Strompreis für die deutsche Industrie um 26 Prozent und für die Haushalte um 23 Prozent angestiegen ist. In Polen belief es sich entsprechend auf 2 bzw. 2,8 Prozent. OSW zitiert die neuesten Untersuchungen, die zeigen, dass im Laufe der letzten drei Jahre der Anteil der Deutschen, der für eine freiwillige Abgabe für den Ausgleich der Treibhausgase ist, um das Dreifache von 3 auf 9 Prozent gestiegen ist. Zirka 20 Prozent der Bevölkerung wollen Strom aus erneuerbaren Energien kaufen (im Jahr 2010 waren es acht Prozent).

Und noch etwas: Interessant sehen vom Standpunkt der polnischen Diskussion über Investitionen für Entwicklung im Lande auch die deutschen Versuche aus, die Energiewende als vorteilhaft für die eigenen wirtschaftlichen Interessen darzustellen. Unter anderem deshalb ist es schwer vorstellbar, dass trotz unvermeidlicher Schwierigkeiten, die Wirtschaft auf emissionsarme Gleise zu stellen, Deutschland die Richtung ändern würde. Nach Jahren konsequenter Arbeit ergeben sich nun enorme Vorteile.

Warum komme ich zu dem Schluss, dass die Polen die Energiewende trotz ihres tiefen Misstrauens aufmerksam betrachten sollten? Hier einige Gründe:

Das eingangs dargestellte Beispiel des energieunabhängigen Campus zeigt, in welche Richtung sich das Interesse von Wissenschaftlern und Ingenieuren in den nächsten Jahrzehnten entwickeln wird. Auf der anderen Seite der Oder, in Reichweite, findet die technologisch-gesellschaftliche Revolution bereits statt, und es wäre gut, alles zu tun, um ihr Nutznießer zu werden. Zweitens: Das Beispiel Deutschlands zeigt, dass man an der grünen Energie verdienen kann. Und zum Dritten: Alles weist darauf hin, dass sich die Klimastrategie der EU in den nächsten Jahren nicht ändern wird. Entgegen den Appellen von Seiten der Gewerkschaften und eines Teils der Politiker ist eine Aufkündigung des Klimapaktes durch Polen nicht möglich. Hört man die Stimmen deutscher Experten (die Geopolitik ist hier rücksichtslos: In Berlin hört man Brüssel besser, in Brüssel ist Berlin besser zu vernehmen), dann lässt sich die Entwicklung der Lage folgendermaßen vorhersehen: Um den strengen ökologischen Kurs aufrechtzuerhalten, wird die Europäische Kommission dahingehend wirken, dass 
der Preis für die Erlaubnis zum $\mathrm{CO}_{2}$-Ausstoß mindestens um das Zweifache steigt. Dabei werden die Länder gewinnen, die in ihrem Energiemix die dafür verantwortlichen Energiequellen reduzieren. Das, was sie in grüne Energie investieren, sparen sie bei den Zahlungen für die Emissionserlaubnis. Polen, das auf Investi- tionen im Bereich Braun- und Steinkohle beharrt, verliert doppelt.

Deshalb lohnt es sich hinzusehen, was bei unserem Nachbarn geschieht.

Übersetzung aus dem Polnischen: Silke Plate

Über den Autor

Michał Olszewski ist Pressesprecher von „ClientEarth«, einer Organisation von Juristen, die sich für den Umweltschutz engagiert, sowie Publizist und Schriftsteller.

\section{Der Energiemix im Vergleich und Erdöl/Erdgas nach Herkunftsländern}

Grafik 1: Primärenergieverbrauch nach Energieträgern in Polen und Deutschland, 2000-2010

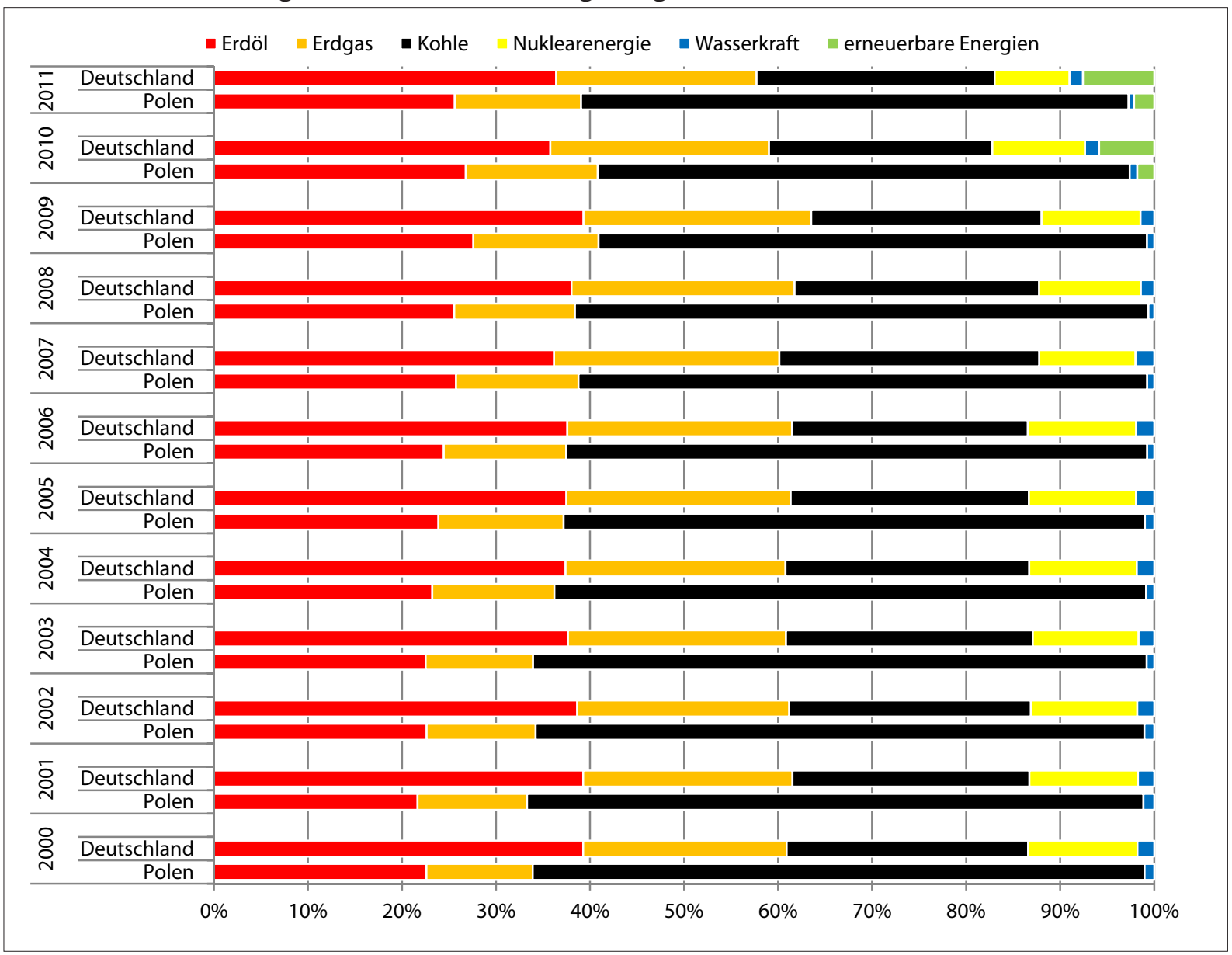

Anm.: Bis 2009 wurde keine Rubrik zu erneuerbaren Energien geführt. Zusammengestellt von Andreas Heinrich, Forschungsstelle Osteuropa an der Universität Bremen.

Quellen: BP Statistical Review of World Energy June 2002; 2003; 2005; 2006; 2007; 2008; 2009; 2010; 2012. 


\begin{tabular}{|c|c|c|c|c|c|c|c|c|c|}
\hline & 8 & 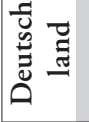 & $\stackrel{\Re}{\widetilde{\Xi}}$ & 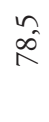 & $\underset{\infty}{\vec{\infty}}$ & $\stackrel{\hat{\sigma}}{\hat{m}}$ & ñ & & $\begin{array}{l}\approx \\
\infty \hat{N} \\
\tilde{m}\end{array}$ \\
\hline & 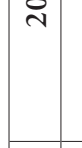 & 䒕 & $\overrightarrow{\widetilde{d}}$ & $\stackrel{\text { }}{\tilde{y}}$ & 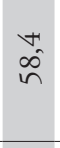 & & $\hat{0}$ & & $\tilde{a}$ \\
\hline ह & 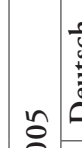 & 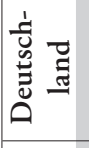 & $\stackrel{n}{\vec{I}}$ & $\stackrel{n}{\wedge}$ & $\overrightarrow{\hat{j}}$ & bे & $\tilde{\sigma}^{n}$ & & $\underset{\sim}{\varpi}$ \\
\hline & $\stackrel{\sim}{2}$ & $\begin{array}{l}\frac{\bar{\Xi}}{0} \\
\stackrel{0}{0}\end{array}$ & $\hat{\vec{v}}$ & Î & $\begin{array}{l}\hat{\emptyset} \\
\varkappa^{\circ}\end{array}$ & & $\hat{o}$ & & $\hat{\vec{a}}$ \\
\hline$\Xi$ & 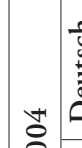 & 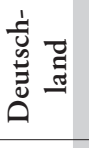 & 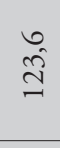 & $\stackrel{2}{\wedge}$ & $\hat{\infty}$ & $\begin{array}{l}\infty \\
\hat{n}\end{array}$ & $\overrightarrow{0}$ & & 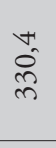 \\
\hline$=$ & 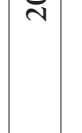 & $\frac{\pi}{0}$ & $\stackrel{m}{\vec{\lambda}}$ & $\Rightarrow$ & $\hat{\hat{n}}$ & & $\begin{array}{l}\infty \\
0 \\
0\end{array}$ & & $\stackrel{\infty}{\hat{a}}$ \\
\hline త్ & $\approx$ & 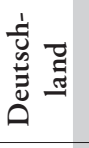 & $\vec{\cong}$ & $\hat{\wedge}$ & $\stackrel{\sim}{\stackrel{\infty}{\infty}}$ & $\begin{array}{l}+ \\
\stackrel{n}{n}\end{array}$ & $\approx$ & & $\begin{array}{l}\overrightarrow{\widetilde{m}} \\
\tilde{n}\end{array}$ \\
\hline 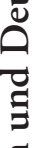 & స & $\frac{\Xi}{0}$ & $\hat{\sigma}$ & $\overrightarrow{0}$ & $\hat{\hat{n}}$ & & $\hat{0}$ & & $\begin{array}{l}\stackrel{n}{\infty} \\
\infty \\
\infty\end{array}$ \\
\hline$\Xi$ & $\check{\Xi}$ & 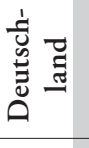 & $\stackrel{\cong}{\cong}$ & $\underset{\stackrel{m}{+}}{\stackrel{m}{N}}$ & $\begin{array}{l}0 \\
\stackrel{+}{\infty}\end{array}$ & $\stackrel{n}{n}$ & $\hat{n}$ & & $\begin{array}{l}\stackrel{\star}{~} \\
\text { ते }\end{array}$ \\
\hline 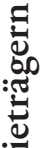 & î & $\frac{\pi}{0}$ & $\hat{\Omega}$ & $\overrightarrow{0}$ & 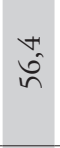 & & $\hat{o}$ & & $\vec{\infty}$ \\
\hline 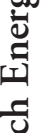 & & 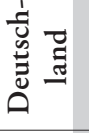 & $\underset{\sim}{\stackrel{0}{m}}$ & 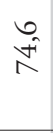 & $\underset{+f}{\stackrel{t}{\infty}}$ & 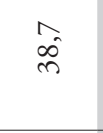 & $\stackrel{\infty}{\dot{n}^{2}}$ & & $\hat{n}$ \\
\hline త্ & & $\frac{\Xi}{0}$ & $\beth$ & 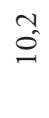 & $\hat{n}$ & & - & & $\hat{\hat{\infty}}$ \\
\hline . & 8 & 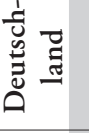 & 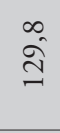 & $\hat{i}$ & $\underset{\infty}{\stackrel{+}{f}}$ & $\stackrel{\forall}{b^{\prime}}$ & $\hat{n}$ & & $\begin{array}{l}n \\
\hat{n} \\
\tilde{n}\end{array}$ \\
\hline ఏ్ & & $\frac{\tilde{0}}{0}$ & $\stackrel{\sim}{\sim}$ & 우 & $\begin{array}{l}0 \\
\hat{n}\end{array}$ & & $\hat{o}$ & & $\begin{array}{l}\vec{t} \\
\infty \\
\infty\end{array}$ \\
\hline$\subseteq$ & & & $\begin{array}{l}\overline{\overline{0}} \\
\overline{\bar{v}} \\
\overline{4}\end{array}$ & 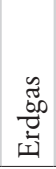 & $\begin{array}{l}\frac{0}{\pi} \\
\frac{\pi}{2}\end{array}$ & 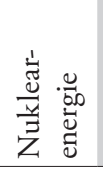 & 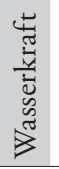 & 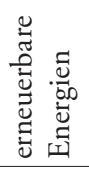 & 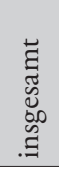 \\
\hline
\end{tabular}

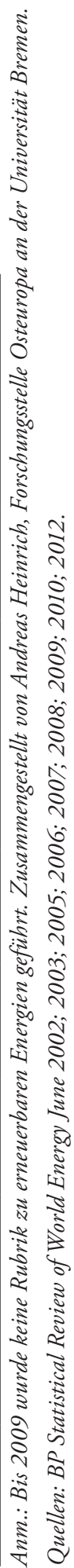


Grafik 2: Polen: Erdgasimporte 1990 und 2010

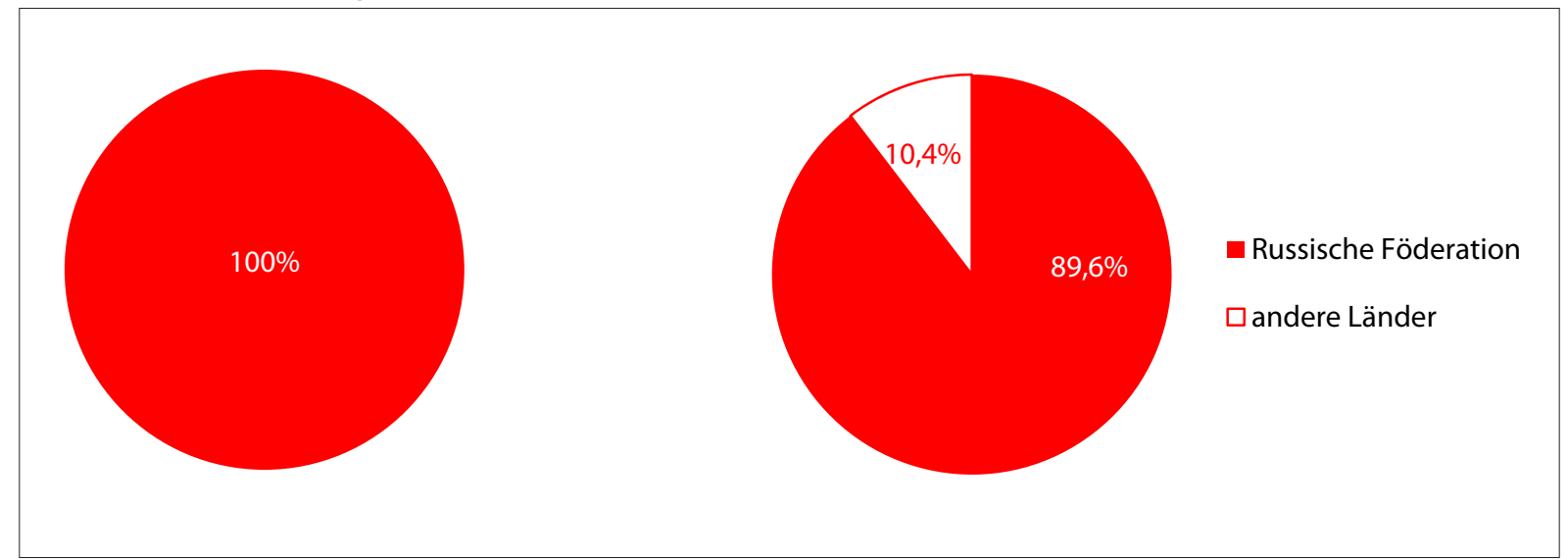

Quelle: Eurostat, http://epp.eurostat.ec.europa.eu/portal/page/portal/statistics/search_database, Zusammengestellt von Andreas Heinrich, Forschungsstelle Osteuropa an der Universität Bremen.

Tabelle 2: Polen: Erdgasimporte insgesamt (in Terajoules) und nach Ländern

\begin{tabular}{|l|r|r|r|r|r|r|r|r|r|r|r|r|c|}
\hline & 1990 & 1995 & $\mathbf{2 0 0 0}$ & $\mathbf{2 0 0 1}$ & $\mathbf{2 0 0 2}$ & $\mathbf{2 0 0 3}$ & $\mathbf{2 0 0 4}$ & $\mathbf{2 0 0 5}$ & $\mathbf{2 0 0 6}$ & $\mathbf{2 0 0 7}$ & $\mathbf{2 0 0 8}$ & $\mathbf{2 0 0 9}$ & $\mathbf{2 0 1 0}$ \\
\hline insgesamt & 315,1 & 217,5 & 308,9 & 335,5 & 312,4 & 350,6 & 379,5 & 398,5 & 415,7 & 385,4 & 425,9 & 379,5 & 414,6 \\
\hline $\begin{array}{l}\text { Russische } \\
\text { Föderation }\end{array}$ & $100 \%$ & $99,1 \%$ & $81,1 \%$ & $83,3 \%$ & $86,1 \%$ & $78,6 \%$ & $62,3 \%$ & $65,9 \%$ & $68,8 \%$ & $67,6 \%$ & $69,4 \%$ & $82,0 \%$ & $89,6 \%$ \\
\hline $\begin{array}{l}\text { Ukraine } \\
\text { Usbekistan }\end{array}$ & & $0,4 \%$ & & & $2,4 \%$ & $3,2 \%$ & $2,9 \%$ & $<0,1 \%$ & $<0,1 \%$ & $<0,1 \%$ & $<0,1 \%$ & $0,1 \%$ & $0,1 \%$ \\
\hline $\begin{array}{l}\text { Turkmenistan } \\
\text { Kasachstan } \\
\text { fühere }\end{array}$ & & & $13,0 \%$ & $8,5 \%$ & & $7,9 \%$ & $25,5 \%$ & $14,4 \%$ & $22,4 \%$ & $23,9 \%$ & & & \\
$\begin{array}{l}\text { Sowjetunion } \\
\text { (unspezifiziert) }\end{array}$ & & & & & & & & $9,1 \%$ & & & & & \\
\hline $\begin{array}{l}\text { Deutschland } \\
\text { Norwegen }\end{array}$ & & $0,5 \%$ & $5,8 \%$ & $4,9 \%$ & $5,2 \%$ & $4,8 \%$ & $4,1 \%$ & $3,5 \%$ & $4,9 \%$ & $8,5 \%$ & $8,1 \%$ & $10,9 \%$ & $10,4 \%$ \\
\hline
\end{tabular}

Quelle: Eurostat, http://epp.eurostat.ec.europa.eu/portal/page/portal/statistics/search_database, Zusammengestellt von Andreas Heinrich, Forschungsstelle Osteuropa an der Universität Bremen. 
Tabelle 3: Polen: Erdölimporte insgesamt (in Tausend Tonnen) und nach Ländern

\begin{tabular}{|c|c|c|c|c|c|c|c|c|c|c|c|c|c|}
\hline & 1990 & 1995 & 2000 & 2001 & 2002 & 2003 & 2004 & 2005 & 2006 & 2007 & 2008 & 2009 & 2010 \\
\hline insgesamt & 13,1 & 12,9 & 18,0 & 17,6 & 17,9 & 17,4 & 17,3 & 17,9 & 19,8 & 20,9 & 20,8 & 20,1 & 22,7 \\
\hline $\begin{array}{l}\text { Russ. Födera- } \\
\text { tion }\end{array}$ & $100 \%$ & $100 \%$ & $93,3 \%$ & $94,7 \%$ & $96,5 \%$ & $95,4 \%$ & $96,3 \%$ & $97,5 \%$ & $96,7 \%$ & $95,6 \%$ & $92,6 \%$ & $94,2 \%$ & $93,1 \%$ \\
\hline Ukraine & & & $0,5 \%$ & $0,4 \%$ & $0,7 \%$ & $2,2 \%$ & $1,9 \%$ & $0,3 \%$ & $0,1 \%$ & $>0,1 \%$ & $>0,1 \%$ & $>0,1 \%$ & \\
\hline Slowakei & & & $0,1 \%$ & $0,1 \%$ & $>0,1 \%$ & $>0,1 \%$ & & & & & & & \\
\hline $\begin{array}{l}\text { Tschechische } \\
\text { Republik }\end{array}$ & & & $0,1 \%$ & $0,1 \%$ & $0,1 \%$ & $>0,1 \%$ & $>0,1 \%$ & $0,1 \%$ & $0,1 \%$ & & & & $>0,1 \%$ \\
\hline Litauen & & & $1,7 \%$ & $2,0 \%$ & $0,2 \%$ & $>0,1 \%$ & & & & & & & \\
\hline Kasachstan & & & $1,6 \%$ & $1,6 \%$ & $0,8 \%$ & $1,0 \%$ & $1,1 \%$ & $0,8 \%$ & $0,2 \%$ & $0,3 \%$ & $0,1 \%$ & $>0,1 \%$ & \\
\hline Aserbaidschan & & & & & & & & & & & $1,0 \%$ & & \\
\hline Deutschland & & & & $0,7 \%$ & $0,6 \%$ & $0,6 \%$ & & & & & & & \\
\hline Niederlande & & & & & $0,2 \%$ & & & & & & & & \\
\hline Norwegen & & & $0,6 \%$ & & $0,3 \%$ & & $0,8 \%$ & $0,7 \%$ & $0,7 \%$ & $2,4 \%$ & $5,5 \%$ & $1,4 \%$ & $5,4 \%$ \\
\hline Schweden & & & & & $0,2 \%$ & & & & & & & & \\
\hline Dänemark & & & & & & & & & & & & & $0,4 \%$ \\
\hline Großbritannien & & & $2,0 \%$ & & $0,4 \%$ & & & $0,5 \%$ & $0,8 \%$ & $1,6 \%$ & $0,8 \%$ & $1,6 \%$ & $0,7 \%$ \\
\hline Kolumbien & & & & & & & & & & & & & $0,4 \%$ \\
\hline Algerien & & & & & & & & & & & & $2,8 \%$ & \\
\hline Kuwait & & & & & & & & & $1,4 \%$ & & & & \\
\hline andere & & & & $0,5 \%$ & & $0,7 \%$ & & & & & & & \\
\hline
\end{tabular}

Quelle: Eurostat, http://epp.eurostat.ec.europa.eu/portal/page/portal/statistics/search_database, Zusammengestellt von Andreas Heinrich, Forschungsstelle Osteuropa an der Universität Bremen. 


\section{Vom 19. März bis zum 15. April 2013}

\begin{tabular}{|c|c|}
\hline 19.03.2013 & $\begin{array}{l}\text { Nach neuesten Angaben des Statistischen Hauptamts (Główny Urząd Statystyczny - GUS) fiel die Industrie- } \\
\text { produktion im Februar im Vergleich zum Vormonat um 2,8\%, bezogen auf Februar } 2012 \text { um 2,1 \%. Die Bau- } \\
\text { wirtschaft wies im Februar } 2013 \text { gegenüber Januar } 2013 \text { ein Minus von 0,3\% und gegenüber Februar } 2012 \text { ein } \\
\text { Minus von } 11,4 \% \text { auf. }\end{array}$ \\
\hline 20.03 .201 & $\begin{array}{l}\text { Außenminister Radosław Sikorski stellt im Sejm die Prioritäten der polnischen Außenpolitik vor. Einen Schwer- } \\
\text { punkt legt er auf die Beziehungen Polens zur Europäischen Union. Angesichts der Distanz Großbritanniens } \\
\text { gegenüber der EU und der instabilen Lage in Südeuropa habe Polen die Chance, Anschluss an den inneren } \\
\text { Entscheidungskreis der EU zu finden. Um seine Bedeutung noch zu erhöhen, müsse Polen der Eurozone bei- } \\
\text { treten. Mit Deutschland teile Polen die strategische Vision der Zukunft und die Methoden zur Behebung der } \\
\text { gegenwärtigen Krise der EU. }\end{array}$ \\
\hline 21.03.2013 & $\begin{array}{l}\text { Die Europäische Kommission teilt mit, dass sie Polen vor den Europäischen Gerichtshof verklagen wird, da die } \\
\text { EU-Richtlinie zu erneuerbaren Energien nicht umgesetzt wurde. }\end{array}$ \\
\hline 22.03 .2013 & $\begin{array}{l}\text { Aus Anlass des Tages der Polnisch-Ungarischen Freundschaft empfängt Staatspräsident Bronisław Komorowski } \\
\text { in Warschau seinen ungarischen Amtskollegen Janos Ader. Beide bekräftigen die Beständigkeit der Freundschaft } \\
\text { und die sehr guten Beziehungen. Am nächsten Tag wird ein eintägiges Polnisch-Ungarisches Wirtschaftsfo- } \\
\text { rum in Tarnów stattfinden. Der Tag der Polnisch-Ungarischen Freundschaft wurde } 2007 \text { vom Sejm und vom } \\
\text { ungarischen Parlament eingesetzt. }\end{array}$ \\
\hline 23.03 .2013 & $\begin{array}{l}\text { Auf einer Sitzung des Landesrats der Bürgerplattform (Platforma Obywatelska - PO) gibt Ministerpräsident } \\
\text { Donald Tusk bekannt, dass er erneut für den Posten des Vorsitzenden der PO kandidieren wird. Für den außer- } \\
\text { ordentlichen Parteitag der PO im Herbst kündigt er eine Abstimmung über die Änderung der Parteistatuten } \\
\text { an. Es soll auch darüber entschieden werden, ob der Parteivorsitzende von den Parteimitgliedern künftig direkt } \\
\text { gewählt werden soll. }\end{array}$ \\
\hline 25.03 .2013 & $\begin{array}{l}\text { Staatspräsident Bronisław Komorowski empfängt in Warschau den Präsidenten des ukrainischen Parlaments, } \\
\text { Wolodymyr Rybak. Thematisiert wird die Perspektive der Unterzeichnung des Assoziationsabkommens der } \\
\text { Ukraine mit der EU anlässlich des Gipfels der Östlichen Partnerschaft im November in Wilna. Komorowski } \\
\text { äußert die Hoffnung, dass die Ukraine die dafür notwendigen Reformen durchführen wird. In der vergange- } \\
\text { nen Woche hatte bereits ein trilaterales Gespräch darüber auch mit dem ungarischen Staatspräsidenten Janos } \\
\text { Ader stattgefunden. }\end{array}$ \\
\hline 26.03 .2013 & $\begin{array}{l}\text { In der Woiwodschaft Schlesien (województwo śląskie) findet ein mehrstündiger Generalstreik aus Protest gegen } \\
\text { die Wirtschafts- und Sozialpolitik der Regierung statt. Schätzungen der Gewerkschaften zufolge nehmen zirka } \\
85.000 \text { Personen teil. Erwartet wurden bis zu } 100.000 \text { Streikende. Gefordert werden u. a. der Verzicht auf Ände- } \\
\text { rungen im Arbeitsrecht, eine Beschränkung prekärer Arbeitsverträge, Unterstützung für die energieintensive } \\
\text { Industrie, Unterstützung für Firmen in der Wirtschaftskrise und Änderungen im Gesundheitssystem. Minis- } \\
\text { terpräsident Donald Tusk unterstreicht, dass u. a. die Forderungen nach einer übermäßigen Erhöhung des Min- } \\
\text { destlohns und nach Verzicht der Flexibilisierung der Arbeitszeit nicht der Schaffung von Arbeitsplätzen dienen. }\end{array}$ \\
\hline 27.03 .2013 & $\begin{array}{l}\text { Das Verfassungsgericht urteilt, dass die Umstrukturierung der Amtsgerichte, die Anfang des Jahres von Justiz- } \\
\text { minister Jarosław Gowin veranlasst worden war, verfassungsgemäß ist. Der Landesrat des Gerichtswesens sowie } \\
\text { Abgeordnete der Polnischen Bauernpartei (Polskie Stronnictwo Ludowe - PSL) hatten in der Maßnahme eine } \\
\text { Einschränkung der Unabhängigkeit des Gerichtswesens gesehen. }\end{array}$ \\
\hline 28.03 .2013 & $\begin{array}{l}\text { Der Botschafter der Republik Polen in Berlin, Jerzy Margański, kritisiert im »ZDF-Morgenmagazin« den drei- } \\
\text { teiligen Fernsehfilm des ZDF „Unsere Mütter, unsere Väter«, der in der vorangegangenen Woche im deutschen } \\
\text { Fernsehen ausgestrahlt worden ist. Damit knüpft er an seinen Brief an das ZDF vom Vortag an. Insbesondere } \\
\text { verweist er auf die ungerechte Akzentuierung der polnischen Heimatarmee (Armia Krajowa-AK) im Film, die } \\
\text { als antisemitisch und geldgierig dargestellt wird. Wichtige historische Ereignisse, wie der Warschauer Aufstand, } \\
\text { die Zerstörung Warschaus und die Hilfe von Polen für Juden, seien nicht beachtet worden. Das ZDF bedauert } \\
\text { in einer Presseerklärung, dass der Film als ungerecht und verletzend empfunden worden sei; die historischen } \\
\text { Tatsachen und die Verantwortung der Deutschen sollten nicht relativiert werden. Deshalb sei der Entstehung } \\
\text { des Films von Fachhistorikern und die Ausstrahlung von historischen Dokumentationen begleitet worden. }\end{array}$ \\
\hline 29.03 .2013 & $\begin{array}{l}\text { Das Finanzministerium teilt mit, dass das Defizit des öffentlichen Haushalts Ende Februar } 2013 \text { 21,68 Mrd. } \\
\text { Zloty betrug. Dies sind } 61 \% \text { des im Haushaltsgesetz für } 2013 \text { genehmigten Defizits. Die Ausgaben beliefen } \\
\text { sich auf } 64,49 \mathrm{Mrd} \text {. Zloty, das sind 19,3\% der geplanten Ausgaben. Die Einnahmen des öffentlichen Haus- } \\
\text { halts in Höhe von 42,8 Mrd. Zloty machten 14,3\% der vorgesehen Einnahmen aus. }\end{array}$ \\
\hline
\end{tabular}




\begin{tabular}{|c|c|}
\hline 31.03 .2013 & $\begin{array}{l}\text { In der Predigt am Ostersonntag führt der Vorsitzende des Polnischen Episkoptas, Erzbischof Józef Michalik, } \\
\text { die wirtschaftliche Krise Europas auf eine Identitätskrise zurück. Ein Europa, das sich nicht zu seinen ethischen } \\
\text { und moralischen Wurzeln auf der Grundlage des Evangeliums bekennen will und einen Bezug zum Christen- } \\
\text { tum aus seiner Verfassung streicht, fördere subjektiven Liberalismus und Egoismus, was zu Krisen unterschied- } \\
\text { licher Art führe. }\end{array}$ \\
\hline 02.04 .2013 & $\begin{array}{l}\text { Nach neuesten Angaben von Eurostat betrug die Arbeitslosigkeit in Polen im Februar 10,6\%, in der Euro- } \\
\text { zone im selben Zeitraum } 12 \% \text {. Dies bedeutet in beiden Fällen keine Veränderungen gegenüber Januar } 2013 .\end{array}$ \\
\hline 03.04 .2013 & $\begin{array}{l}\text { Der stellvertretende Außenminister Artur Nowak-Far stellt in einer Sitzung des Auswärtigen Ausschusses des } \\
\text { Sejm die Situation des Weimarer Dreiecks (Deutschland, Frankreich, Polen) dar. Seit } 2010 \text { belebe sich die } \\
\text { Zusammenarbeit, die Polen nutze wolle, um als Interessenvertreter Ostmitteleuropas aufzutreten. Thematisiert } \\
\text { werden sollen die finanzielle Situation und die Sicherheits- und Verteidigungspolitik der EU. Das Weimarer } \\
\text { Dreieck solle gegen Tendenzen, die EU zu schwächen, auftreten, so die Auffassung des Außenministeriums. }\end{array}$ \\
\hline 04.04 .2013 & $\begin{array}{l}\text { In einem Interview für Tageszeitungen aus Tschechien, Ungarn und der Slowakei unterstreicht Außenminis- } \\
\text { ter Radosław Sikorski die Bedeutung der Zusammenarbeit der Visegrád-Gruppe (Polen, Tschechien, Slowa- } \\
\text { kei, Ungarn) innerhalb der EU. Die vier Länder hätten dieselbe Sicht u. a. auf Fragen des Straßenbaus und der } \\
\text { Energieinfrastruktur. Außerdem bestünde Übereinstimmung darin, dass die Geschichte dieser Region Euro- } \\
\text { pas vor westlicher Ignoranz geschützt werden müsse. }\end{array}$ \\
\hline 05.04 .2013 & $\begin{array}{l}\text { Der Sejm lehnt ein Misstrauensvotum gegen Transportminister Sławomir Nowak mit } 228 \text { zu } 220 \text { Stimmen ab. } \\
\text { Der Misstrauensantrag wurde von Solidarisches Polen von Zbigniew Ziobro (Solidarna Polska Zbigniewa Zio- } \\
\text { bra) gestellt und von der Demokratischen Linksallianz (Sojusz Lewicy Demokratycznej - SLD) und Recht und } \\
\text { Gerechtigkeit (Prawo i Sprawiedliwość - PiS) unterstützt. Nowak wird u. a. vorgeworfen, dass der Zustand der } \\
\text { Straßen und des Schienennetzes schlecht sei und EU-Finanzmitteln falsch eingesetzt würden. }\end{array}$ \\
\hline 06.04 . & $\begin{array}{l}\text { Nach Ansicht des stellvertretenden Ministerpräsidenten und Wirtschaftsministers Janusz Piechociński handelt } \\
\text { es sich bei dem am Vortag von den Energiekonzernen Gazprom und EuRoPol Gaz unterzeichneten Memoran- } \\
\text { dum über die Möglichkeiten, einen Abzweig der Gaspipeline Jamal II von Belarus durch Polen in die Slowakei } \\
\text { und nach Ungarn zu führen, um interne Machtspiele des russischen Gassektors auf verschiedenen Ebenen, die } \\
\text { auch in die Politik des Kreml hineinreichen würden. In Polen entzündet sich in diesem Zusammenhang eine } \\
\text { Diskussion über die Energiesicherheit Polens und Energieunabhängigkeit von Russland. }\end{array}$ \\
\hline 07.04 & $\begin{array}{l}\text { Staatspräsident Bronisław Komorowski begrüßt die Begnadigung des ehemaligen Innenministers der Ukraine, } \\
\text { Jurij Luzenko, eines Vertrauten der inhaftierten ehemaligen Regierungschefin Julia Timoschenko, durch den } \\
\text { ukrainischen Staatspräsidenten Wiktor Janukowitsch. Dieser Schritt diene der Verbesserung des Image der } \\
\text { Ukraine, was neben notwendigen Reformen die Bedingung für die Unterzeichnung des Assoziierungsabkom- } \\
\text { mens zwischen der EU und der Ukraine sei. }\end{array}$ \\
\hline 08.04 .2013 & $\begin{array}{l}\text { Das Parteigericht der Demokratischen Linksallianz (Sojusz Lewicy Demokratycznej - SLD) schließt Ryszard } \\
\text { Kalisz aus der Partei aus. Hintergrund ist das Engagement des früheren SLD-Justizministers für die politische } \\
\text { Bewegung »Europa Plus«, an der u. a. die Palikot-Bewegung (Ruch Palikota) beteiligt ist. }\end{array}$ \\
\hline 09.04 .2013 & $\begin{array}{l}\text { Nach einer Kabinettssitzung kündigt Ministerpräsident Donald Tusk an, dass die Diskussion über die Offe- } \\
\text { nen Rentenfonds (Otwarte Fundusze Emerytalne - OFE) im Juni abgeschlossen werden soll. Hintergrund ist } \\
\text { das Inkrafttreten von EU-Bestimmungen, nach denen die OFE die Möglichkeit hätten, an ausländischen Bör- } \\
\text { sen zu investieren. Die OFE sind eine der drei Säulen des polnischen Rentenversicherungssystems. Tusk versi- } \\
\text { chert, dass die staatliche Sozialversicherungsanstalt (Zakład Ubezpieczeń Społecznych - ZUS) und der Staat } \\
\text { die Garanten einer sicheren Rente seien. }\end{array}$ \\
\hline 10.04 .2013 & $\begin{array}{l}\text { Ministerpräsident Donald Tusk trifft zu einem dreitägigen Besuch in Nigeria in der Hauptstadt Abuja ein. Vor- } \\
\text { gesehen sind Treffen mit dem nigerianischen Staats- und Regierungschef Jonathan Goodluck und Gouverneu- } \\
\text { ren sowie die Teilnahme am Nigerianisch-Polnischen Wirtschaftsforum, an dem zirka } 30 \text { polnische Firmen aus } \\
\text { den Bereichen Energie, Waffen, Transport, Lebensmittel und Finanzen teilnehmen. Nigeria ist nach der Repu- } \\
\text { blik Südafrika der zweitgrößte Wirtschaftspartner Polens in Afrika. }\end{array}$ \\
\hline 11.04 .2013 & $\begin{array}{l}\text { Staatspräsident Bronisław Komorowski verlängert auf Antrag der Regierung den Einsatz des polnischen Mili- } \\
\text { tärkontingents im Rahmen der ISAF-Truppen in Afghanistan bis Mitte Oktober 2013. Die polnische Armee } \\
\text { ist in der Provinz Ghazni stationiert, wo seit dem 1. April die afghanische Armee und Polizei die Verantwor- } \\
\text { tung für die Sicherheit übernommen haben. Seit } 2007 \text { sind } 39 \text { Angehörige des polnischen Militärkontingents } \\
\text { in Afghanistan ums Leben gekommen. }\end{array}$ \\
\hline 12.04 .2013 & $\begin{array}{l}\text { Staatspräsident Bronisław Komorowski unterzeichnet die Gesetzesnovelle zur technischen Modernisierung und } \\
\text { Finanzierung der Streitkräfte. Diese beinhaltet auch die Finanzierung eines polnischen Raketenabwehrprogramms. }\end{array}$ \\
\hline
\end{tabular}




\begin{tabular}{|l|l|}
\hline 13.04.2013 & $\begin{array}{l}\text { In Warschau treffen sich die Parlamentsvorsitzenden der Länder der Visegrád-Gruppe (Polen, Slowakei, Tsche- } \\
\text { chien, Ungarn). Verabschiedet wurde eine gemeinsame Erklärung über eine verstärkte Zusammenarbeit der Par- } \\
\text { lamente und den Aufbau einer starken Region, um in der EU mehr Gehör zu finden, so Sejmmarschallin Ewa } \\
\text { Kopacz. Ein wichtiges Gesprächsthema auf dem Treffen war die Wirtschaftskrise der EU. }\end{array}$ \\
\hline 15.04.2013 & $\begin{array}{l}\text { Ryszard Kalisz, ehemaliger Politiker der Demokratischen Linksallianz (Sojusz Lewicy Demokratycznej - } \\
\text { SLD), kündigt an, bis Ende des Monats eine eigene politische Vereinigung zu gründen, die mit der Wahlliste } \\
\text { von »Europa Plus« für die Wahl zum Europäischen Parlament zusammenarbeiten wird. Kalisz war Anfang der } \\
\text { Woche aus der SLD aufgrund seines Engagements für »Europa Plus« ausgeschlossen worden. }\end{array}$ \\
\hline
\end{tabular}

Sie können die gesamte Chronik seit 2007 auch auf http://www.laender-analysen.de/polen/ unter dem Link»Chronik« lesen. 


\section{Dritte Tagung Deutsche Polenforschung Gießen, 20.-22. März 2014 Wissen, Verstehen, Übersetzen: Nachbarn im Dialog Call for Papers / Call for Panels}

Gute Nachbarschaft ist anspruchsvoll: Sie erfordert Wahrnehmung, Respekt, Verständnis und Verständigung, gemeinsame Regeln, Normen und vielfältiges Wissen voneinander. Werden Befindlichkeiten und Bedürfnisse des Anderen nicht bemerkt, mangelt es an Wissen, Verstehen, Übersetzen, so entstehen Missverständnisse und Konflikte. Polens Nachbarschaftsbeziehungen verdeutlichen dies exemplarisch, in der Geschichte ebenso wie in der Gegenwart. Dabei ist nicht nur an das polnisch-deutsche Verhältnis zu denken, sondern auch an die zahlreichen anderen Nachbarn im Norden, Süden und Osten sowie an die "fremden Nachbarn« im Lande selbst, insbesondere an die jüdische Bevölkerung.

Die Dritte Tagung Deutsche Polenforschung ist Forum für Wissenschaftlerinnen und Wissenschaftler aller Disziplinen, die sich inhaltlich mit Polen beschäftigen. Das Rahmenthema ist bewusst so gewählt, dass viele verschiedene Fächer Ansatzpunkte finden: Zu fragen ist etwa nach sprachlicher Übersetzung und literarischer Transmission, nach kulturellen, technischen oder rechtlichen Transfers, nach sozialen und ökonomischen Begegnungsräumen, Konzeptwanderung oder Erfahrungen von Verflechtung und Entflechtung in Politik, Gesellschaft, Kultur und Wirtschaft. Das Rahmenthema wird in Sektionen, Podiumsdiskussionen, Werkstattgesprächen, Einzelvorträgen und Projektvorstellungen mit seinen unterschiedlichen historischen und aktuellen Aspekten diskutiert werden.

Neben den geistes- und kulturwissenschaftlichen Fächern sollen auf der Dritten Tagung Deutsche Polenforschung auch die sozial- und wirtschaftswissenschaftlichen Disziplinen stärker berücksichtigt werden. Beiträge aus den Gebieten Soziologie, Politikwissenschaft oder Wirtschaftswissenschaft sind deshalb besonders willkommen.

Die Dritte Tagung Deutsche Polenforschung bietet die Gelegenheit, über die Grenzen der Fachgebiete und der deutschsprachigen Länder hinweg Kontakte zu knüpfen und zu pflegen, Projekte zu entwickeln und sich über die Situation der deutschen Polenforschung zu informieren. Sie knüpft an die beiden ersten Tagungen (Darmstadt 2009, Mainz 2011) an, an denen jeweils weit mehr als 200 Wissenschaftlerinnen und Wissenschaftlern teilgenommen hatten. Ausstellungen von Verlagen und Institutionen sowie ein Begleitprogramm ergänzen die Tagung. Tagungssprache ist Deutsch, in Ausnahmefällen sind Englisch oder Polnisch zugelassen.

\section{Vorbereitungsteam der Tagung}

Prof. Dr. Dieter Bingen (Darmstadt), Prof. Dr. Hans-Jürgen Bömelburg (Gießen), Prof. Dr. Thomas Daiber (Gießen), Prof. Dr. Alfred Gall (Mainz), Prof. Dr. Peter Haslinger (Marburg/Gießen), Prof. Dr. Jan Kusber (Mainz), Dr. Peter Oliver Loew (Darmstadt), Prof. Dr. Monika Wingender (Gießen), Prof. Dr. Klaus Ziemer (Trier).

\section{Call for Papers oder Panels}

Wir laden alle Interessierten ein, Vorschläge für Referate oder ganze Sektionen einzureichen, die sich am Rahmenthema "Wissen, Verstehen, Übersetzen: Nachbarn im Dialog« orientieren sollten; es können auch Vorschläge mit einem anderen thematischen Schwerpunkt eingereicht werden, wenn sie von besonderer Forschungsrelevanz sind.

Einzelvorträge: Die Dauer von Einzelvorträgen ist auf 25 Minuten begrenzt. Die Organisatoren werden die ausgewählten Einzelvorträge zu Sektionen zusammenfassen.

Sektionsvorschläge: Die Sektionsdauer beträgt 2 1/4 Stunden. Die Zahl der Referenten sollte drei bis vier betragen; es wird empfohlen, jeweils einen Kommentar vorzusehen. Sektionen mit Vertretern jeweils nur eines Wissenschaftsstandorts sind ausgeschlossen. Die Veranstalter behalten sich das Recht vor, die ausgewählten Sektionen in Absprache mit den Vorschlagenden zu ergänzen oder zu modifizieren.

Bewerbungsschluss für Papers und Panels ist der 15. Juni 2013. Über die Auswahl wird bis Ende September 2013 entschieden.

\section{Projektvorstellungen}

Zusätzlich zu den längeren Referaten bieten wir - in erster Linie für Nachwuchswissenschaftler/-innen - die Möglichkeit zu kurzen Projektvorstellungen im Plenum (2-3 Minuten). Hierzu melden Sie sich bitte bis 15. Dezember 2013 an. Über die Auswahl der Vorstellungen wird bis Ende Januar 2014 entschieden. 
Posterpräsentationen

Wenn Sie Ihr Projekt mit einem Poster präsentieren möchten, so können Sie diese bis 31. Januar 2014 anmelden.

\section{Ausstellungen, Präsentationen}

Aussteller (Institutionen, Verlage, Unternehmen) können sich in unterschiedlicher Art und Weise auf der Dritten Tagung Deutsche Polenforschung präsentieren. Für die Anmietung von Möbeln (Tische, Stühle, Stellwände) entstehen voraussichtlich geringe Kosten. Sollten Sie Interesse haben, so bitten wir um Ihre Abstimmung mit den Veranstaltern bis 15. Dezember 2013.

\section{Einladung zur Teilnahme ohne eigenen Beitrag}

Alle an einer Teilnahme ohne eigenen Beitrag Interessierten laden wir ein, sich auf unserer Homepage www.polenfor schung.de anzumelden.

\section{Für alle Teilnehmer (Referierende, Teilnehmerinnen und Teilnehmer)}

Wir bitten alle Teilnehmer, ob mit oder ohne Referat, sich über unsere Tagungshomepage www.polenforschung.de anzumelden. Sie reisen und übernachten auf Ihre Kosten. Der Teilnehmerbeitrag beläuft sich auf 35 Euro (Studierende, Doktoranden, Arbeitslose 25 Euro, Spätanmeldegebühr ab 1.2.2014 60 Euro).

Bei einer Anmeldung bis 1. Februar 2014 wird ein Tagungsbeitrag in Höhe von 35 Euro erhoben (Studierende, Doktoranden, Arbeitslose 25 Euro), danach wird eine Spätanmeldegebühr von 60 Euro fällig. Die Kosten für Pausengetränke und für den Abendempfang am 20. März 2014 werden von den Veranstaltern übernommen.

Die Organisatoren werden Ihnen ab Herbst 2013 bei der Suche nach Übernachtungsmöglichkeiten behilflich sein; es werden Zimmerkontingente in Gießener Hotels vorgehalten. Alle angemeldeten Personen erhalten rechtzeitig weitere Informationen.

\section{Anmeldungen}

Einreichung von Vorschlägen für Vorträge und Projektvorstellungen sowie Ansprechpartner für alle inhaltlichen Fragen: Dr. Peter Oliver Loew, Deutsches Polen-Institut, E-Mail: loew@dpi-da.de, Tel. +49/6151/4202-17, Fax: +49/6151/4202-10.

Anmeldung von Posterpräsentationen, Ständen, Ausstellungen sowie Ansprechpartnerin für alle organisatorischen und allgemeinen Fragen: Iwona Maj-Mehrvarz, Deutsches Polen-Institut, E-Mail: maj-mehrvarz@dpi-da.de, Tel.: +49/6151/4202-11, Fax: Fax: +49/6151/4202-10

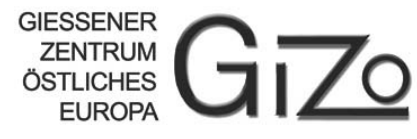

DEUTSCHES POLEN INSTITUT

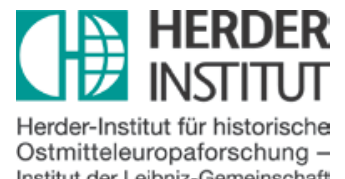

Ostmitteleuropaforschung - 
Die Polen-Analysen erscheinen zweimal monatlich als E-Mail-Dienst. Sie werden gemeinsam vom Deutschen PolenInstitut Darmstadt, der Bremer Forschungsstelle Osteuropa und der Deutschen Gesellschaft für Osteuropakunde in Partnerschaft mit dem Willy Brandt Zentrum für Deutschland- und Europastudien an der Universität Wroclaw (Breslau) herausgegeben.

Ein Archiv der Polen-Analysen finden Sie im Internet unter www.laender-analysen.de/polen

Kostenloses Abonnement unter http://www.deutsches-polen-institut.de/Newsletter/subscribe.php

Diese Analysen finden Sie online als Lizenzausgabe auf bpb.de

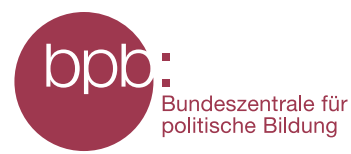

\section{Deutsches Polen-Institut Darmstadt}

Das Deutsche Polen-Institut Darmstadt (DPI) ist ein Forschungs-, Informations-, und Veranstaltungszentrum für polnische Kultur, Geschichte, Politik, Gesellschaft und die deutsch-polnischen Beziehungen, die sich im Kontext der europäischen Integration entwickeln. Das seit März 1980 aktive und bis 1997 von Gründungsdirektor Karl Dedecius geleitete Institut ist eine Gemeinschaftsgründung der Stadt Darmstadt, der Länder Hessen und Rheinland-Pfalz sowie des Bundes. 1987 wurden die Kultusminister der Länder und 2011 das Auswärtige Amt weitere institutionelle Träger. Einen wesentlichen Beitrag zur Verwirklichung der Institutsziele leisten private Stiftungen. Das DPI hat satzungsgemäß die Aufgabe, durch seine Arbeit zur Vertiefung der gegenseitigen Kenntnisse des kulturellen, geistigen und gesellschaftlichen Lebens von Polen und Deutschen beizutragen.

Ziel der Vermittlertätigkeit des DPI ist es, „die zu interessieren, auf die es politisch, wirtschaftlich, gesellschaftlich und kulturell im deutsch-polnischen Verhältnis ankommt (Leitlinien 1997). Es geht um die Entscheider und Multiplikatoren in Politik, Kultur, Bildung, Verwaltung, Medien und Wirtschaft und, wesentlich stärker ausgeprägt als bisher, um das Hineinwirken in Wissenschaft, Forschung und Bildung.

Derzeit bemüht sich das DPI in Kooperation mit den verstreuten Orten wissenschaftlicher Polen-Kompetenz an deutschen Hochschulen und Forschungsinstituten verstärkt darum, ausgehend von einer Bestandsaufnahme deutscher Polen-Forschung Ort wissenschaftlicher Forschung und verbindendes, vernetzendes und kooperierendes Zentrum zu werden. Ausgangspunkt der Neuausrichtung ist die kaum mehr kontrollierbare Dynamik des Rückbaus der Ressourcen der wissenschaftlichen Polen-Kompetenz in den unterschiedlichen Disziplinen. Mit der über 60.000 Bände zählenden multidisziplinären Fachbibliothek für Polen, die eine einzigartige Sammlung polnischer Literatur in der Originalsprache und in deutscher Übersetzung umfasst, ist das DPI bereits ein geschätzter Ort der Recherche und des wissenschaftlichen Arbeitens. (www.deutsches-polen-institut.de)

\section{Forschungsstelle Osteuropa an der Universität Bremen (www.forschungsstelle.uni-bremen.de)}

1982 gegründet, widmet sich die Forschungsstelle Osteuropa an der Universität Bremen der interdisziplinären Analyse der Länder Ost- und Ostmitteleuropas in Zeitgeschichte und Gegenwart. Der Forschungsschwerpunkt liegt dabei auf der Rolle von »Dissens und Konsens«, von Opposition und Zivilgesellschaft in ihrem historischen, politischen, gesellschaftlichen und kulturellen Kontext. Die Forschungsstelle besitzt in ihrem Archiv eine einzigartige Sammlung alternativer Kulturgüter und unabhängiger Texte aus den ehemaligen sozialistischen Ländern. Darunter befindet sich auch eine umfangreiche Sammlung des "Zweiten Umlaufs«, die das Schrifttum und Dokumente unabhängiger Initiativen und gesellschaftlicher Gruppen in Polen aus der Zeit von 1976 bis zum Umbruch umfasst. Hinzu kommt eine umfangreiche Bibliothek mit wissenschaftlicher Literatur. Mit Archiv, Bibliothek und zwei wissenschaftlichen Abteilungen ist die Forschungsstelle auch eine Anlaufstelle sowohl für Gastwissenschaftler als auch für die interessierte Öffentlichkeit.

Eine der Hauptaufgaben der Forschungsstelle ist die Information der interessierten Öffentlichkeit. Dazu gehören unter anderem regelmäßige E-Mail-Informationsdienste für Politik, Wirtschaft, Zivilgesellschaft und Medien.

\section{Herausgegeben mit finanzieller Unterstützung der Stiftung für deutsch-polnische Zusammenarbeit}

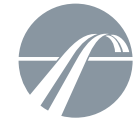

FUNDACJA WSPÓEPRACY

STIFTUNG

STIFTUNG
FUR DEUTSCH-POLNISCHE
ZUSAMMENARBEIT

Die Meinungen, die in den Polen-Analysen geäußert werden, geben ausschließlich die Auffassung der Autoren wieder.

Abdruck und sonstige publizistische Nutzung sind nach Rücksprache mit der Redaktion gestattet.

Redaktion: Prof. Dr. Dieter Bingen (verantwortlich) (Darmstadt), Silke Plate, M.A. (Bremen) Technische Gestaltung: Matthias Neumann

Polen-Analysen-Layout: Cengiz Kibaroglu, Matthias Neumann

Alle Ausgaben der Polen-Analysen sind mit Themen- und Autorenindex archiviert unter www.laender-analysen.de

Die Polen-Analysen werden im Rahmen der Datenbank World Affairs Online (WAO) ausgewertet und sind im Portal IREON www.ireon-portal.de recherchierbar.

ISSN 1863-9712 @ 2013 by Deutsches Polen-Institut Darmstadt und Forschungsstelle Osteuropa, Bremen

Kontakt: Dr. Andrzej Kaluza, Presse- und Öffentlichkeitsarbeit, Deutsches Polen-Institut, Mathildenhöhweg 2,

D-64287 Darmstadt, Tel.: 06151/4985-13, Fax: 06151/4985-10, E-Mail: polen-analysen@dpi-da.de, Internet: www.laender-analysen.de/polen 